Stud. Univ. Babeş-Bolyai Math. 62(2017), No. 4, 413-426

DOI: 10.24193/subbmath.2017.4.01

\title{
Quadratic refinements of matrix means
}

\author{
Mohammad Sababheh
}

\begin{abstract}
The main target of this article is to present refinements of the matrix arithmetic-geometric mean inequality. The main difference between these refinements and the ones in the literature is the quadratic behavior of the refining terms. These refinements include the Löewner partial ordering, determinants, trace and unitarily invariant norms refinements.
\end{abstract}

Mathematics Subject Classification (2010): 15A39, 15B48, 47A30, 47A63.

Keywords: Positive matrices, matrix means, norm inequalities, Young's inequality.

\section{Introduction and motivation}

Let $\mathbb{M}_{n}$ be the algebra of $n \times n$ complex matrices, $\mathbb{M}_{n}^{+}$be the cone of positive semidefinite matrices in $\mathbb{M}_{n}$ and $\mathbb{M}_{n}^{++}$be the cone of strictly positive matrices in $\mathbb{M}_{n}$. For two Hermitian matrices $A$ and $B$, we write $A \geq B$ or $B \leq A$ to mean $A-B \in \mathbb{M}_{n}^{+}$, while we write $A>B$ or $B<A$ to mean $A-B \in \mathbb{M}_{n}^{++}$.

Comparison between Hermitian matrices is receiving a considerable attention these days, where the possible comparison between the means of these matrices is extensively considered.

In this article, we compare between matrices using the partial ordering $\leq$ defined above and using invariant norms. Recall that a norm $\left\||\||\right.$ on $\mathbb{M}_{n}$ is called invariant, if $\left\|\left|U A V\left\|\left|=\left\||A \||\right.\right.\right.\right.\right.$ for all $A \in \mathbb{M}_{n}$ and all unitary matrices $U, V$. Among the most useful invariant norms, is the Hilbert-Schmidt norm \|\|$_{2}$ defined as follows

$$
\|A\|_{2}=\left(\sum_{i, j=1}^{n}\left|a_{i j}\right|^{2}\right)^{\frac{1}{2}}, A=\left[a_{i j}\right] .
$$

Notice that this is equivalent to $\|A\|_{2}=\sqrt{\operatorname{tr}|A|^{2}}$, where $|A|^{2}=A^{*} A$ and $\operatorname{tr}$ is the trace functional. 
Another possible comparison between matrices is the comparison between their determinants, where the identity $\operatorname{det} A=\prod_{i=1}^{n} \lambda_{i}(A)$ becomes handy. In this context, $\left\{\lambda_{i}(A)\right\}$ refers to the set of eigenvalues of $A$.

Further, for $A, B \in \mathbb{M}_{n}^{++}$and $0 \leq t \leq 1$, we define the weighted arithmetic and geometric means, respectively, as follows

$$
A \nabla_{t} B=(1-t) A+t B \text { and } A \#_{t} B=A^{\frac{1}{2}}\left(A^{-\frac{1}{2}} B A^{-\frac{1}{2}}\right)^{t} A^{\frac{1}{2}} .
$$

When $t=\frac{1}{2}$, it is customary to drop it from the notation. So we write $A \nabla B$ to denote $A \nabla_{\frac{1}{2}} B$, for example. Among the most well established comparisons between matrices is the following inequality known as the "arithmetic-geometric mean inequality"

$$
A \#_{t} B \leq A \nabla_{t} B, 0 \leq t \leq 1 .
$$

Then this means' inequality is refined and reversed in many ways. Before stating these refinements, we remind the reader that obtaining such matrix inequalities is done in different ways, but the most common technique is by a corresponding numerical inequality. For this, we need to look at the numerical forms of the above inequality. For the positive numbers $a, b$ and $0 \leq t \leq 1$, we define the weighted means by $a \nabla_{t} b=(1-t) a+t b$ and $a \#_{t} b=a^{1-t} b^{t}$. The above matrix mean inequality can be simply proved using the known numerical inequality

$$
a \#_{t} b \leq a \nabla_{t} b, 0 \leq t \leq 1 .
$$

This inequality is well known by Young's inequality. We explain how to move from a numerical inequality to a matrix inequality in Theorem 2.10 below.

Since the matrix versions are obtained from numerical versions, refinements and reverses of numerical inequalities imply certain refinements and reverses of matrix inequalities. We mention here a few refinements known in the literature. In [5] it is proved that

$$
a \#_{t} b+\min \{t, 1-t\}(\sqrt{a}-\sqrt{b})^{2} \leq a \nabla_{t} b
$$

or simply

$$
a \#_{t} b+L_{1}(t) f_{1}(a, b) \leq a \nabla_{t} b
$$

for a piecewise linear function $L_{1}$ and some positive function $f_{1}(a, b)$. On the other hand, a two-term refinement has been proved in [16]

$$
a \#_{t} b+L_{1}(t) f_{1}(a, b)+L_{2}(t) f_{2}(a, b) \leq a \nabla_{t} b,
$$

for another piecewise linear function $L_{2}$ and another positive function $f_{2}$. These refinements then were generalized in [13], [14] as

$$
a \#_{t} b+\sum_{i=1}^{N} L_{i}(t) f_{i}(a, b) \leq a \nabla_{t} b, N \in \mathbb{N}
$$

for piecewise linear functions $L_{i}$ and positive functions $f_{i}$.

Moreover, the reversed version

$$
a \nabla_{t} b \leq a \#_{t} b+\max \{t, 1-t\}(\sqrt{a}-\sqrt{b})^{2}, 0 \leq t \leq 1
$$

was proved in [6], and a generalization was presented recently in [13].

Further related results can be found in [1], [5], [12], [15], [16], [17]. 
What is common among these different refinements and reverses is the fact that all refining terms are linear in $t$.

In the recent paper [8], a quadratic refinement and reverse of Young's inequality were proved. Namely, it is shown that if $a, b>0$ and $0 \leq t \leq 1$ are such that $(b-a)(2 t-1) \geq 0$ then

$$
a \#_{t} b+2 t(1-t)(\sqrt{a}-\sqrt{b})^{2} \leq a \nabla_{t} b
$$

while we have the reversed inequality if $(b-a)(2 t-1) \leq 0$. Notice that the refining term in this inequality is $2 t(1-t)(\sqrt{a}-\sqrt{b})^{2}$ which is quadratic in $t$.

Our motivation of the current work begins with this observation. In fact, even (1.3) follows from a more general quadratic refinement. Our main target in this paper is to show that certain quadratic refinements and reverses can be shown for the arithmetic-geometric mean inequality, in both multiplicative and additive forms. Among many other matrix versions, we prove the following inequalities for $A, B \in \mathbb{M}_{n}^{++}$and $X \in \mathbb{M}_{n}$ under some ordering condition,

$$
\begin{gathered}
\tau(1-\tau)\left(A \nabla_{\nu} B-A \#_{\nu} B\right) \leq \nu(1-\nu)\left(A \nabla_{\tau} B-A \#_{\tau} B\right) \\
\operatorname{det}\left(A \#_{\nu} B\right)^{\frac{1}{n}}+\frac{\nu(1-\nu)}{\tau(1-\tau)} \operatorname{det}\left(A \nabla_{\tau} B-A \#_{\tau} B\right)^{\frac{1}{n}} \leq \operatorname{det}\left(A \nabla_{\nu} B\right)^{\frac{1}{n}} \\
\operatorname{tr}\left|A^{1-\nu} B^{\nu}\right|+\frac{\nu(1-\nu)}{\tau(1-\tau)}\left(\operatorname{tr}\left(A \nabla_{t} B\right)-\operatorname{tr} A \#{ }_{\tau} \operatorname{tr} B\right) \leq \operatorname{tr}\left(A \nabla_{\nu} B\right)
\end{gathered}
$$

and

$$
\frac{\|(1-\nu) A X+\nu X B\|_{2}^{2}-\left\|A^{\nu} X B^{1-\nu}\right\|_{2}^{2}}{\nu(1-\nu)} \leq \frac{\|(1-\tau) A X+\tau X B\|_{2}^{2}-\left\|A^{\tau} X B^{1-\tau}\right\|_{2}^{2}}{\tau(1-\tau)} .
$$

A common aspect of all the refinements in this paper is the quadratic refining term $\nu(1-\nu)$ or $\tau(1-\tau)$.

We remark that in the recent paper [7], quadratic refinements of Heinz inequality have been shown.

The organization of this paper will be as follows. In the first part, we prove the needed numerical inequalities, and these will be done by some calculus computations. Then we present the matrix versions in the same order of the numerical ones to make it easier for the reader to follow.

\section{Main results}

Our main results section is divided into two parts. The first part will treat numerical versions needed to accomplish the matrix versions presented in the second part of the section. 


\subsection{The numerical versions}

In the following computations, the reader must be careful about moving from one variable to another.

Lemma 2.1. For $c>0$, let

$$
f(t)=\frac{1 \nabla_{t} c-1 \#_{t} c}{t(1-t)}, 0<t<1 .
$$

Then

1. $f$ is increasing on $(0,1)$ if $c>1$.

2. $f$ is decreasing on $(0,1)$ if $c<1$.

Proof. Direct computations show that

$$
f^{\prime}(t)=\frac{g(c)}{t^{2}(1-t)^{2}},
$$

where, considering $t$ as a constant,

$$
g(c)=-1+c^{t}(1-2 t)+2 t+(c-1) t^{2}+c^{t}(t-1) t \log c .
$$

Then

$$
g^{\prime}(c)=t^{2} h(c), \text { where } h(c)=1-c^{t-1}(1+(1-t) \log c) .
$$

Furthermore,

$$
h^{\prime}(c)=(1-t)^{2} c^{t-2} \log c .
$$

Now if $c>1$, then $h^{\prime}(c)>0$ while $h^{\prime}(c)<0$ when $c<1$. Therefore $h=h(c)$ attains its minimum at $c=1$. That is $h(c) \geq h(1)=0$. Consequently, $g^{\prime}(c)>0$ and $g(c)$ is increasing on $(0, \infty)$.

If $c>1$ then $g(c) \geq g(1)=0$ and $f^{\prime}(t)>0$. This proves the first statement.

On the other hand, if $c<1, g(c) \leq g(1)=0$ and $f^{\prime}(t)<0$. This proves the second statement.

This entails the following quadratic refinement and reverse of Young's inequality.

Proposition 2.2. Let $a, b>0$ and $0 \leq \nu, \tau \leq 1$. If $(b-a)(\tau-\nu) \geq 0$, then

$$
\tau(1-\tau)\left(a \nabla_{\nu} b-a \#{ }_{\nu} b\right) \leq \nu(1-\nu)\left(a \nabla_{\tau} b-a \#_{\tau} b\right) .
$$

On the other hand, if $(b-a)(\tau-\nu) \leq 0$ then

$$
\tau(1-\tau)\left(a \nabla_{\nu} b-a \#_{\nu} b\right) \geq \nu(1-\nu)\left(a \nabla_{\tau} b-a \#_{\tau} b\right) .
$$

Proof. Letting $c=\frac{b}{a}$ in the function $f(t)=\frac{1 \nabla_{t} c-1 \#_{t} c}{t(1-t)}$ and using the monotonicity of Lemma 2.1 imply both inequalities.

Letting $\nu=\frac{1}{2}$ in the above proposition implies the following [8].

Corollary 2.3. Let $a, b>0$ and $0 \leq \nu \leq 1$. If $(b-a)\left(\tau-\frac{1}{2}\right) \geq 0$ then

$$
a \#_{\tau} b+4 \tau(1-\tau)(a \nabla b-a \# b) \leq a \nabla_{\tau} b .
$$

On the other hand, if $(b-a)\left(\tau-\frac{1}{2}\right) \leq 0$ then

$$
a \#{ }_{\tau} b+4 \tau(1-\tau)(a \nabla b-a \# b) \geq a \nabla_{\tau} b .
$$


This explains the generality of these inequalities. Then squared versions that we can use to prove some Hilbert-Schmidt norm forms can be obtained as follows.

Proposition 2.4. For $c>0$, define $f:(0,1) \rightarrow[0, \infty)$ by

$$
f(t)=\frac{\left(1 \nabla_{t} c\right)^{2}-\left(1 \#_{t} c\right)^{2}}{t(1-t)} .
$$

1. If $c<1$, then $f$ is decreasing on $(0,1)$ and

2. if $c>1$, then $f$ is increasing on $(0,1)$.

Proof. Direct computations show that

$$
f^{\prime}(t)=\frac{g(c)}{t^{2}(1-t)^{2}}, g(c)=-1+2 t-t^{2}+c^{2} t^{2}+c^{2 t}(1-2 t+2(-1+t) t \log c) .
$$

Further,

and

$$
g^{\prime}(c)=\frac{2 t^{2}}{c} h(t), h(t)=c^{2}+c^{2 t}(-1+2(-1+t) \log c)
$$

$$
h^{\prime}(t)=4 c^{2 t}(t-1) \log ^{2} c .
$$

Clearly $h^{\prime}(t)<0$, hence $h(t) \geq h(1)=0$ and $g^{\prime}(c) \geq 0$. If $c<1$, then $g(c) \leq$ $g(1)=0$ and $f$ is decreasing. On the other hand, if $c>1, g(c) \geq g(1)=0$ and $f$ is increasing.

Corollary 2.5. Let $a, b>0$ and $0<\nu, \tau<1$. If $(b-a)(\tau-\nu) \geq 0$ then

$$
\frac{\left(a \nabla_{\nu} b\right)^{2}-\left(a \#_{\nu} b\right)^{2}}{\nu(1-\nu)} \leq \frac{\left(a \nabla_{\tau} b\right)^{2}-\left(a \#_{\tau} b\right)^{2}}{\tau(1-\tau)} .
$$

The inequality is reversed if $(b-a)(\tau-\nu) \leq 0$.

Again, letting $\tau=\frac{1}{2}$, we obtain the corresponding inequality from [8].

The above two refinements are "additive" versions, where the refining term is added to one side of the original inequality. Our next result presents a multiplicative form of these inequalities.

Lemma 2.6. For $c>0$, define $f:(0,1) \rightarrow[0, \infty)$ by

$$
f(t)=\left(\frac{1 \nabla_{t} c}{1 \#_{t} c}\right)^{\frac{1}{t(1-t)}}
$$

Then

1. $f$ is increasing on $(0,1)$ if $c<1$ and

2. $f$ is decreasing on $(0,1)$ if $c>1$.

Proof. Let $F(t)=\log f(t)$. That is

$$
F(t)=\frac{\log (1-t+t c)-t \log c}{t(1-t)} .
$$

Then

$$
F^{\prime}(t)=\frac{g(c)}{(1-t)^{2} t^{2}(1-t+t c)}
$$


where

$$
g(c)=t(c-1+t-t c+t(t-1-t c) \log c)+(2 t-1)(1-t+t c) \log (1-t+t c) .
$$

Now

$$
g^{\prime}(c)=-\frac{t}{c} h(c) \text { for } h(c)=(c-1)(t-1) t+c t^{2} \log c+c(1-2 t) \log (1-t+t c) .
$$

Furthermore,

$$
h^{\prime}(c)=t^{2} \log c+(2 t-1)\left[\frac{(c-1)(t-1) t}{1-t+t c}-\log (1-t+t c)\right]
$$

and

$$
h^{\prime \prime}(c)=\frac{(1-t)^{2} t\left(2 c+(c-1)^{2} t\right)}{c(1-t+t c)^{2}} .
$$

Now clearly $h^{\prime \prime}(c) \geq 0$, hence $h^{\prime}$ is increasing in $c$.

If $c<1$, then $h^{\prime}(c) \leq h^{\prime}(1)=0$ and $h$ is decreasing when $c \leq 1$. That is, $h(c) \geq h(1)=0$ and $g^{\prime}(c) \leq 0$ when $c \leq 1$. Thus, $g$ is decreasing when $c \leq 1$, and hence $g(c) \geq g(1)=0$. Consequently, $F^{\prime}(t) \geq 0$ and $F$ is increasing in $t$, when $c \leq 1$. This proves the first assertion. When $c>1$, a similar argument implies that $F$ is decreasing in $t$.

As a consequence, we obtain the following multiplicative refinement and reverse of Young's inequality.

Corollary 2.7. Let $a, b>0$ and $0<\nu, \tau<1$. If $(b-a)(\tau-\nu)>0$ then

$$
a \#_{\tau} b\left(\frac{a \nabla_{\nu} b}{a \#_{\nu} b}\right)^{\frac{\tau(1-\tau)}{\nu(1-\nu)}} \geq a \nabla_{\tau} b .
$$

On the other hand, if $(b-a)(\tau-\nu)<0$ then

$$
a \#_{\tau} b\left(\frac{a \nabla_{\nu} b}{a \#_{\nu} b}\right)^{\frac{\tau(1-\tau)}{\nu(1-\nu)}} \leq a \nabla_{\tau} b .
$$

Proof. Let $c=\frac{b}{a}$ in

$$
f(t)=\left(\frac{1 \nabla_{t} c}{1 \#_{t} c}\right)^{\frac{1}{t(1-t)}} .
$$

If $b<a$, then $f$ is increasing, by Lemma 2.6. Therefore, when $\nu<\tau$ we have $f(\nu) \leq$ $f(\tau)$. This completes the proof of the first inequality. A similar argument implies the second inequality.

Remark 2.8. Having introduced our numerical quadratic refinements and reverses, we compare these results with the linear inequalities. We have seen that, for $a, b>0$ and $0 \leq t \leq 1$, one has

$$
r(t)(\sqrt{a}-\sqrt{b})^{2} \leq a \nabla_{t} b-a \#_{t} b \leq R(t)(\sqrt{a}-\sqrt{b})^{2},
$$

where $r(t)=\min \{t, 1-t\}$ and $R(t)=\max \{t, 1-t\}$. On the other hand, under certain ordering conditions, we have the quadratic refinement or reverse

$$
a \nabla_{t} b-a \#_{t} b \leq(\geq) 2 t(1-t)(\sqrt{a}-\sqrt{b})^{2} .
$$


It is natural to ask about the advantage of introducing a quadratic refinement or reverse over the linear ones.

Direct calculations show that, for $0 \leq t \leq 1$, one has $r(t) \leq 2 t(1-t)$ and $R(t) \geq$ $2 t(1-t)$. Therefore, when $(b-a)(2 t-1) \geq 0$, we have

$$
a \#_{t} b+r(t)(\sqrt{b}-\sqrt{a})^{2} \leq a \#_{t} b+2 t(1-t)(\sqrt{b}-\sqrt{a})^{2} \leq a \nabla_{t} b,
$$

which is a refinement of the refinement (1.1). On the other hand, if $(b-a)(2 t-1) \leq 0$, we have the

$$
a \#_{t} b+R(t)(\sqrt{b}-\sqrt{a})^{2} \geq a \#_{t} b+2 t(1-t)(\sqrt{b}-\sqrt{a})^{2} \geq a \nabla_{t} b,
$$

which is a refinement of the reversed version (1.2). Therefore, introducing quadratic refinements serves as introducing one-term refinements of the already existing linear refinements.

A similar argument applies for the multiplicative versions.

We conclude this section by the following observation. The inequalities in Proposition 2.2 and Corollary 2.5 give rise to the following quotients

$$
\frac{a \nabla_{\nu} b-a \#_{\nu} b}{a \nabla_{\tau} b-a \#_{\tau} b} \text { and } \frac{\left(a \nabla_{\nu} b\right)^{2}-\left(a \#_{\nu} b\right)^{2}}{\left(a \nabla_{\tau} b\right)^{2}-\left(a \#_{\tau} b\right)^{2}} .
$$

It is natural to ask about the relation between these quantities. Denoting these quotients by $A_{\nu, \tau}(a, b)$ and $A_{\nu, \tau}^{(2)}(a, b)$, respectively, we have the following comparison.

Proposition 2.9. Let $a, b>0$ and $0 \leq \nu, \tau \leq 1$. If $(b-a)(\tau-\nu) \geq 0$, then

$$
A_{\nu, \tau}^{(2)}(a, b) \leq A_{\nu, \tau}(a, b)
$$

On the other hand, if $(b-a)(\tau-\nu) \leq 0$, then

$$
A_{\nu, \tau}^{(2)}(a, b) \geq A_{\nu, \tau}(a, b)
$$

Proof. Let $f(t)=a \nabla_{t} b+a \#_{t} b$. Then, clearly, $f$ is increasing when $b>a$ and is decreasing if $b<a$. Now, if $(b-a)(\tau-\nu) \geq 0$, then $f(\tau) \geq f(\nu)$, whether $b>a$ or $b<a$. Simplifying the inequality $f(\nu) \leq f(\tau)$ implies the inequality $A_{\nu, \tau}^{(2)}(a, b) \leq A_{\nu, \tau}(a, b)$, when $(b-a)(\tau-\nu) \geq 0$. A similar argument implies the reversed inequality when $(b-a)(\tau-\nu) \leq 0$.

\subsection{Matrix versions}

Now we present the matrix versions one can obtain from the numerical versions proved above.

Theorem 2.10. Let $A, B \in \mathbb{M}_{n}^{++}$and $0 \leq \nu, \tau \leq 1$. If $(\tau-\nu)(B-A) \geq 0$ then

$$
\tau(1-\tau)\left(A \nabla_{\nu} B-A \#_{\nu} B\right) \leq \nu(1-\nu)\left(A \nabla_{\tau} B-A \#_{\tau} B\right) .
$$

The inequality is reversed if $(\tau-\nu)(B-A) \leq 0$.

Proof. If $(\tau-\nu)(B-A) \geq 0$, let $X=A^{-\frac{1}{2}} B A^{-\frac{1}{2}}$. Notice that if $\tau>\nu$ then $B \geq A$ and $\lambda_{i}(X) \geq 1, \forall i$. That is, $(\tau-\nu)\left(\lambda_{i}(X)-1\right) \geq 0$. A similar conclusion is achieved 
if $\tau<\nu$. Now since $(\tau-\nu)\left(\lambda_{i}(X)-1\right) \geq 0$, we may apply the first inequality of Proposition 2.2, using $a=1$ and $b=\lambda_{i}(X)$. This implies

$$
\tau(1-\tau)\left(1 \nabla_{\nu} \lambda_{i}(X)-1 \#_{\nu} \lambda_{i}(X)\right) \leq \nu(1-\nu)\left(1 \nabla_{\tau} \lambda_{i}(X)-1 \#_{\tau} \lambda_{i}(X)\right),
$$

which implies

$$
\begin{aligned}
\tau(1-\tau) & \left(I_{n} \nabla_{\nu} \operatorname{diag}\left(\lambda_{i}(X)\right)-I_{n} \#{ }_{\nu} \operatorname{diag}\left(\lambda_{i}(X)\right)\right) \\
& \leq \nu(1-\nu)\left(I_{n} \nabla_{\tau} \operatorname{diag}\left(\lambda_{i}(X)\right)-I_{n} \#_{\tau} \operatorname{diag}\left(\lambda_{i}(X)\right)\right) .
\end{aligned}
$$

Now since $X$ is Hermitian, $X=U \operatorname{diag}\left(\lambda_{i}(X)\right) U^{*}$ for some unitary matrix $U$. Conjugating the above inequality by $U$ and noticing that conjugation is order preserving, we get

$$
\tau(1-\tau)\left(I_{n} \nabla_{\nu} X-I_{n} \#{ }_{\nu} X\right) \leq \nu(1-\nu)\left(I_{n} \nabla_{\tau} X-I_{n} \#_{\tau} X\right) .
$$

Now conjugating this inequality with $A^{\frac{1}{2}}$ implies the first desired inequality. The second inequality follows similarly.

On the other hand, a determinant version may be obtained as follows. First, we recall Minkowski inequality, [3], p. 560,

$$
\left(\prod_{i=1}^{n} a_{i}\right)^{\frac{1}{n}}+\left(\prod_{i=1}^{n} b_{i}\right)^{\frac{1}{n}} \leq\left(\prod_{i=1}^{n}\left(a_{i}+b_{i}\right)\right)^{\frac{1}{n}}
$$

for the positive numbers $\left\{a_{i}, b_{i}: 1 \leq i \leq n\right\}$.

Theorem 2.11. Let $A, B \in \mathbb{M}_{n}^{++}$and $0<\nu, \tau<1$. If $(\tau-\nu)(B-A) \leq 0$ then

$$
\operatorname{det}\left(A \#_{\nu} B\right)^{\frac{1}{n}}+\frac{\nu(1-\nu)}{\tau(1-\tau)} \operatorname{det}\left(A \nabla_{\tau} B-A \#_{\tau} B\right)^{\frac{1}{n}} \leq \operatorname{det}\left(A \nabla_{\nu} B\right)^{\frac{1}{n}} .
$$

Proof. Let $X=A^{-\frac{1}{2}} B A^{-\frac{1}{2}}$. If $(\tau-\nu)(B-A) \leq 0$ then $(\tau-\nu)\left(\lambda_{i}(X)-1\right) \leq 0$, which justifies the application of the second inequality of Proposition 2.2 in the following computations. Now

$$
\begin{aligned}
\operatorname{det}\left(I_{n} \nabla_{\nu} X\right)^{\frac{1}{n}} & =\prod_{i=1}^{n} \lambda_{i}\left((1-\nu) I_{n}+\nu X\right)^{\frac{1}{n}} \\
& \left.=\prod_{i=1}^{n}\left(1-\nu+\nu \lambda_{i}(X)\right)^{\frac{1}{n}} \text { (now apply Proposition } 2.2 \text { then }(2.1)\right) \\
& \geq \prod_{i=1}^{n}\left(\frac{\nu(1-\nu)}{\tau(1-\tau)}\left(1 \nabla_{\tau} \lambda_{i}(X)-1 \#_{\tau} \lambda_{i}(X)\right)+1 \#_{\nu} \lambda_{i}(X)\right)^{\frac{1}{n}} \\
& \geq \prod_{i=1}^{n}\left(\frac{\nu(1-\nu)}{\tau(1-\tau)}\left(1 \nabla_{\tau} \lambda_{i}(X)-1 \#_{\tau} \lambda_{i}(X)\right)\right)^{\frac{1}{n}}+\prod_{i=1}^{n}\left(1 \#_{\nu} \lambda_{i}(X)\right)^{\frac{1}{n}} \\
& =\frac{\nu(1-\nu)}{\tau(1-\tau)} \prod_{i=1}^{n} \lambda_{i}^{\frac{1}{n}}\left(I_{n} \nabla_{\tau} X-I_{n} \#_{\tau} X\right)+\prod_{i=1}^{\frac{1}{n}}\left(I_{n} \#{ }_{\nu} X\right) .
\end{aligned}
$$


Now multiplying both sides with $\operatorname{det} A^{\frac{1}{2}}$ and using basic properties of the determinant imply the desired inequality.

Notice that if we set $\tau=\frac{1}{2}$ in $(2.2)$, we get

$$
\operatorname{det}\left(A \#{ }_{\nu} B\right)^{\frac{1}{n}}+4 \nu(1-\nu) \operatorname{det}(A \nabla B-A \# B)^{\frac{1}{n}} \leq \operatorname{det}\left(A \nabla_{\nu} B\right)^{\frac{1}{n}},
$$

when $(1-2 \nu)(B-A) \leq 0$. Raising both sides to the power $n$ implies

$$
\begin{aligned}
\operatorname{det}\left(A^{1-\nu} B^{\nu}\right) & +4^{n} \nu^{n}(1-\nu)^{n} \operatorname{det}(A \nabla B-A \# B) \\
& \leq\left(\operatorname{det}\left(A \#{ }_{\nu} B\right)^{\frac{1}{n}}+4 \nu(1-\nu) \operatorname{det}(A \nabla B-A \# B)^{\frac{1}{n}}\right)^{n} \\
& \leq \operatorname{det}((1-\nu) A+\nu B) .
\end{aligned}
$$

In [8], it is proved that

$$
\operatorname{det}\left(A^{1-\nu} B^{\nu}\right)+4^{n} \nu^{n}(1-\nu)^{n} \operatorname{det}(A \nabla B-A \# B) \leq \operatorname{det}((1-\nu) A+\nu B) .
$$

Therefore, (2.2) provides a refinement and a generalization of the corresponding result in this reference. On the other hand, Proposition 2.5 maybe used to obtain squared determinant versions as follows.

Proposition 2.12. Let $A, B \in \mathbb{M}_{n}^{++}$and let $0<\nu, \tau<1$. If $(\tau-\nu)(B-A) \geq 0$, then

$$
\operatorname{det}\left(A \#_{\nu} B\right)^{\frac{2}{n}}+\frac{\nu(1-\nu)}{\tau(1-\tau)} \operatorname{det}\left(A \nabla_{\tau} B-A \#_{\tau} B\right)^{\frac{2}{n}} \leq \operatorname{det}\left(A \nabla_{\nu} B\right)^{\frac{2}{n}} .
$$

Proof. Following the same notations of Theorem 2.11, we have

$$
\begin{aligned}
\operatorname{det}\left(I_{n} \nabla_{\nu} X\right)^{\frac{2}{n}} & \left.=\left(\prod_{i=1}^{n}\left(1 \nabla_{\nu} \lambda_{i}(X)\right)^{2}\right)^{\frac{1}{n}} \quad \text { apply Proposition } 2.5 \text { then }(2.1)\right) \\
& \geq \prod_{i=1}^{n}\left(\left(1 \#_{\nu} \lambda_{i}(X)\right)^{2}+\frac{\nu(1-\nu)}{\tau(1-\tau)}\left[\left(1 \nabla_{\tau} \lambda_{i}(X)\right)^{2}-\left(1 \#_{\tau} \lambda_{i}(X)\right)^{2}\right]\right)^{\frac{1}{n}} \\
& \geq \prod_{i=1}^{n}\left(\left(1 \#_{\nu} \lambda_{i}(X)\right)^{2}\right)^{\frac{1}{n}}+\prod_{i=1}^{n}\left(\frac{\nu(1-\nu)}{\tau(1-\tau)}\left[\left(1 \nabla_{\tau} \lambda_{i}(X)\right)^{2}-\left(1 \# \lambda_{\tau}(X)\right)^{2}\right]\right)^{\frac{1}{n}} \\
& \geq \prod_{i=1}^{n}\left(\lambda_{i}\left(I_{n} \#{ }_{\nu} X\right)\right)^{\frac{2}{n}}+\frac{\nu(1-\nu)}{\tau(1-\tau)} \prod_{i=1}^{n}\left(1 \nabla_{\tau} \lambda_{i}(X)-1 \#_{\tau} \lambda_{i}(X)\right)^{\frac{2}{n}} \\
& =\operatorname{det}\left(I_{n} \#{ }_{\nu} X\right)^{\frac{2}{n}}+\frac{\nu(1-\nu)}{\tau(1-\tau)}\left[\operatorname{det}\left(I_{n} \nabla_{\tau} X\right)-\operatorname{det}\left(I_{n} \#{ }_{\tau} X\right)\right]^{\frac{2}{n}}
\end{aligned}
$$

where we have used the simple inequality $\left(a^{2}-b^{2}\right) \geq(a-b)^{2}$ when $a>b>0$ to obtain the last inequality in the above proof. Now multiplying the last inequality with $\operatorname{det} A$ implies the desired inequality.

In the following result, we use the well known inequality [4]

$$
\operatorname{tr}\left|A^{1-\nu} B^{\nu}\right| \leq(\operatorname{tr} A)^{1-\nu}(\operatorname{tr} B)^{\nu}, A, B \in \mathbb{M}_{n}^{+} .
$$


Proposition 2.13. Let $A, B \in \mathbb{M}_{n}^{++}$and let $0<\nu, \tau<1$. If $(\tau-\nu)(\operatorname{tr} B-\operatorname{tr} A) \leq 0$, then

$$
\operatorname{tr}\left|A^{1-\nu} B^{\nu}\right|+\frac{\nu(1-\nu)}{\tau(1-\tau)}\left(\operatorname{tr}\left(A \nabla_{t} B\right)-\operatorname{tr} A \#_{\tau} \operatorname{tr} B\right) \leq \operatorname{tr}\left(A \nabla_{\nu} B\right) .
$$

Proof. Under the condition $(\tau-\nu)(\operatorname{tr} B-\operatorname{tr} A) \leq 0$, we have

$$
\begin{aligned}
\operatorname{tr}\left(A \nabla_{\nu} B\right) & =\operatorname{tr} A \nabla_{\nu} \operatorname{tr} B \\
& \geq \frac{\nu(1-\nu)}{\tau(1-\tau)}\left(\operatorname{tr} A \nabla_{\tau} \operatorname{tr} B-\operatorname{tr} A \#_{\tau} \operatorname{tr} B\right)+\operatorname{tr} A \#_{\nu} \operatorname{tr} B \\
& \geq \operatorname{tr}\left|A^{1-\nu} B^{\nu}\right|+\frac{\nu(1-\nu)}{\tau(1-\tau)}\left(\operatorname{tr}\left(A \nabla_{t} B\right)-\operatorname{tr} A \#_{\tau} \operatorname{tr} B\right),
\end{aligned}
$$

where we have used (2.3) to obtain the last inequality and used Proposition 2.2 to obtain the first inequality.

On the other hand, the squared version in Proposition 2.5 entails the following Hilbert-Schmidt norm inequality. For the next result, $\left\{\lambda_{i}\right\}$ will denote the eigenvalues of $A$ arranged in a decreasing order and $\left\{\mu_{j}\right\}$ will denote the eigenvalues of $B$ arranged in a decreasing order too. Moreover, the notation $X \circ Y$ will mean the Schur product of $X$ and $Y$.

Theorem 2.14. Let $A, B \in \mathbb{M}_{n}^{+}$and $X \in \mathbb{M}_{n}$. If $\tau>\nu$ and $B \geq \lambda_{1} I_{n}$, or if $\tau<\nu$ and $B \leq \lambda_{n} I_{n}$ then

$$
\frac{\|(1-\nu) A X+\nu X B\|_{2}^{2}-\left\|A^{\nu} X B^{1-\nu}\right\|_{2}^{2}}{\nu(1-\nu)} \leq \frac{\|(1-\tau) A X+\tau X B\|_{2}^{2}-\left\|A^{\tau} X B^{1-\tau}\right\|_{2}^{2}}{\tau(1-\tau)} \text {. }
$$

The inequality is reversed if $\tau>\nu$ and $B \leq \lambda_{n} I_{n}$ or if $\tau<\nu$ and $B \geq \lambda_{1} I_{n}$.

Proof. Since $A, B \in \mathbb{M}_{n}^{+}$, there exist unitary matrices $U, V$ and nonnegative numbers $\lambda_{i}, \mu_{j}$ such that

$$
A=U \operatorname{diag}\left(\lambda_{i}\right) U^{*} \text { and } B=V \operatorname{diag}\left(\mu_{j}\right) V^{*} .
$$

Letting $Y=U^{*} X V$, we have

$$
(1-\nu) A X+\nu X B=U\left(\left[(1-\nu) \lambda_{i}+\nu \mu_{j}\right] \circ Y\right) V^{*} .
$$

Notice that the condition $B \geq \lambda_{1} I_{n}$ implies $\mu_{j} \geq \lambda_{i}, \forall i, j$ and the condition $B \leq \lambda_{n} I_{n}$ implies $\mu_{j} \leq \lambda_{i}, \forall i, j$. Therefore, the conditions $\tau>\nu$ and $B \geq \lambda_{1} I_{n}$, or if $\tau<\nu$ and $B \leq \lambda_{n} I_{n}$ imply $(\tau-\nu)\left(\mu_{j}-\lambda_{i}\right) \geq 0, \forall i, j$. Under these conditions, and noting that \|\|$_{2}$ is unitarily invariant, we have

$$
\begin{aligned}
& \|(1-\nu) A X+\nu X B\|_{2}^{2} \\
& =\sum_{i, j}\left\{\left(\lambda_{i} \nabla_{\nu} \mu_{j}\right)^{2}\left|y_{i j}\right|^{2}\right\} \quad \text { (now apply Corollary 2.5) } \\
& \leq \sum_{i, j}\left\{\left(\lambda_{i} \# \nu \mu_{j}\right)^{2}+\frac{\nu(1-\nu)}{\tau(1-\tau)}\left(\left(\lambda_{i} \nabla_{\tau} \mu_{j}\right)^{2}-\left(\lambda_{i} \#{ }_{\tau} \mu_{j}\right)^{2}\right)\right\}\left|y_{i j}\right|^{2} \\
& =\left\|A^{1-\nu} X B^{\nu}\right\|_{2}^{2}+\frac{\nu(1-\nu)}{\tau(1-\tau)}\left(\|(1-\tau) A X+\tau X B\|_{2}^{2}-\left\|A^{1-\tau} X B^{\tau}\right\|_{2}^{2}\right),
\end{aligned}
$$


which completes the proof for the first set of conditions. A similar argument implies the reversed inequality for the other conditions.

Notice that the above inequalities provide a refinement and a reverse of the inequality $\left\|A^{1-\tau} X B^{\tau}\right\|_{2} \leq\|(1-\tau) A X+\tau X B\|_{2}$.

Next, we present a matrix version of Corollary 2.7. For this result, we adopt the notation of Theorem 2.14.

Theorem 2.15. Let $A, B \in \mathbb{M}_{n}^{++}, X \in \mathbb{M}_{n}, 0<\nu, \tau<1$ and let $m, M$ be two positive numbers such that $m I_{n} \leq A, B \leq M I_{n}$. If $\tau>\nu$ and $B \geq \lambda_{1} I_{n}$, or if $\tau<\nu$ and $B \leq \lambda_{n} I_{n}$ then

$$
\left\|A^{1-\tau} X B^{\tau}\right\|_{2}^{2} \geq\left(\frac{m}{M}\right)^{\frac{2 \tau(1-\tau)}{\nu(1-\nu)}}\|(1-\tau) A X+\tau X B\|_{2}^{2} .
$$

Proof. Adopting the notation of Theorem 2.14, notice that the condition $m I_{n} \leq A$, $B \leq M I_{n}$ implies $m \leq \lambda_{i}, \mu_{j} \leq M$ and hence

$$
\frac{m}{M} \leq \frac{\lambda_{i} \#{ }_{\nu} \mu_{j}}{\lambda_{i} \nabla_{\nu} \mu_{j}} \leq \frac{M}{m}, \forall i, j .
$$

Furthermore, the conditions $\tau>\nu$ and $B \geq \lambda_{1} I_{n}$, or if $\tau<\nu$ and $B \leq \lambda_{n} I_{n}$ imply that $\left(\mu_{j}-\lambda_{i}\right)(\tau-\nu) \geq 0, \forall i, j$. Therefore, applying Corollary 2.7 we have

$$
\begin{aligned}
\left\|A^{1-\tau} X B^{\tau}\right\|_{2}^{2} & =\sum_{i, j}\left(\lambda_{i} \#_{\tau} \mu_{j}\right)^{2}\left|y_{i j}\right|^{2} \\
& \geq \sum_{i, j}\left(\frac{\lambda_{i} \#{ }_{\nu} \mu_{j}}{\lambda_{i} \nabla_{\nu} \mu_{j}}\right)^{\frac{2 \tau(1-\tau)}{\nu(1-\nu)}}\left(\lambda_{i} \nabla_{\tau} \mu_{j}\right)^{2}\left|y_{i j}\right|^{2} \\
& \geq\left(\frac{m}{M}\right)^{\frac{2 \tau(1-\tau)}{\nu(1-\nu)}} \sum_{i, j}\left(\lambda_{i} \nabla_{\tau} \mu_{j}\right)^{2}\left|y_{i j}\right|^{2} \\
& =\left(\frac{m}{M}\right)^{\frac{2 \tau(1-\tau)}{\nu(1-\nu)}}\|(1-\tau) A X+\tau X B\|_{2}^{2} .
\end{aligned}
$$

This completes the proof.

Notice that Theorem 2.15 provides a reverse of the well known inequality $\left\|A^{1-\tau} X B^{\tau}\right\|_{2} \leq\|(1-\tau) A X+\tau X B\|_{2}$. Further, notice that the condition $B \geq \lambda_{1} I_{n}$ means that $B \geq\|A\| I_{n}$ where $\|A\|$ is the operator norm, while the condition $B \leq \lambda_{n} I_{n}$ means that $A \geq\|B\| I_{n}$.

On the other hand, unitarily invariant norm inequalities can be obtained as follows. Recall first that for $A, B \in \mathbb{M}_{n}^{++}$and $X \in \mathbb{M}_{n}$, we have the well known Hölder inequality [4]

$$
\left\|\left|A ^ { 1 - t } X B ^ { t } \left\|\left|\leq\left\|\left|A X\left\|^{1-t}\right\|\right| X B\right\|^{t}, 0 \leq t \leq 1,\right.\right.\right.\right.
$$

for any unitarily invariant norm $\left\||\||\right.$ on $\mathbb{M}_{n}$. Applying Young's inequality on the left side implies the known matrix Young inequality

$$
\left\|\left|A^{1-t} X B^{t}\||\leq(1-t)\||A X\||+t\|\mid X B\| \|\right.\right.
$$


We remark that the inequality $\left\|\left|A^{1-t} X B^{t}\||\leq\||(1-t) A X+t X B \||\right.\right.$ is not true in general, however, it is true for the norm \|\|$_{2}$.

In [11], it has been shown that the function $f(t)=\left\|\left|A^{1-t} X B^{t} \|\right|, 0 \leq t \leq 1\right.$ is log-convex. We use this fact to present the following reverse of (2.4).

Lemma 2.16. Let $A, B \in \mathbb{M}_{n}^{++}, X \in \mathbb{M}_{n}$ and $\||\||$ be a unitarily invariant norm on $\mathbb{M}_{n}$ such that $\left\|\mid A^{1-t} X B^{t}\right\| \| \neq 0$ for any $0 \leq t \leq 1$. Then

$$
\left\|\left|A ^ { 1 - t } X B ^ { t } \left\|\left|\left(\frac{\||A X\||\||X B \||}{\left\|\left|A^{\frac{1}{2}} X B^{\frac{1}{2}} \|\right|^{2}\right.}\right)^{R(t)} \geq\left\|\left|A X\left\|^{1-t}\right\|\right| X B\right\|^{t},\right.\right.\right.\right.
$$

where $R(t)=\max \{t, 1-t\}$.

Proof. Let $f(t)=\left\|\mid A^{1-t} X B^{t}\right\| \|$. Then $f$ is log-convex. For $0 \leq t \leq \frac{1}{2}$, notice that

$$
\frac{1}{2}=\alpha t+(1-\alpha) \text { where } \alpha=\frac{1}{2-2 t} .
$$

Using log-convexity of $f$, we have

$$
f\left(\frac{1}{2}\right) \leq f^{\alpha}(t) f^{1-\alpha}(1)
$$

Simplifying this inequality implies the result for $0 \leq t \leq \frac{1}{2}$. Similar computations yield the result for $\frac{1}{2} \leq t \leq 1$.

On the other hand, notice that the function $f(t)=\||(1-t) A X+t X B \||$ is convex. This fact follows immediately because $\||\||$ is a norm. This entails the following reverse of $\||(1-t) A X+t X B\||\leq(1-t)\||A X\||+t\||X B \||$. The proof is similar to the above one. However, the reader is encouraged to look at [10] for a general discussion of these refinements and reverses of convex functions.

Lemma 2.17. Let $A, B \in \mathbb{M}_{n}^{++}, X \in \mathbb{M}_{n}$ and $\||\||$ be a unitarily invariant norm on $\mathbb{M}_{n}$. Then

$$
\begin{aligned}
\|\mid(1-t) A X+t X B\| & +R(t)(\||A X\||+\||X B\||-\||A X+X B \||) \\
& \leq(1-t)\||A X\||+t\||X B \|| .
\end{aligned}
$$

Now we are ready to find quadratic refinements and reverses of

$$
\left\|\left|A ^ { 1 - t } X B ^ { t } \left\|\left|\leq\left\|\left|A X\left\|\left.\right|^{1-t}\right\|\right| X B\right\|^{t} \leq(1-t)\||A X\||+t\|\mid X B\| \| .\right.\right.\right.\right.
$$

Theorem 2.18. Let $A, B \in \mathbb{M}_{n}^{++}, X \in \mathbb{M}_{n}$ and $\||\||$ be a unitarily invariant norm on $\mathbb{M}_{n}$ such that $\left\|\left|A^{1-t} X B^{t} \|\right| \neq 0\right.$ for any $0 \leq t \leq 1$. If $(\||X B\||-\||A X \||)(\tau-\nu)>0$ then

$$
\begin{aligned}
& \||(1-\tau) A X+\tau X B \|| \\
& \leq(1-\tau)\||A X\||+\tau\||X B \|| \\
& \leq\left.\left\|\left|A X\left\|\left.\right|^{1-\tau}\right\|\right| X B\right\|\right|^{\tau}\left(\frac{(1-\nu)\||A X\||+\nu\||X B \||}{\left.\left\|\left|A X\left\|\left.\right|^{1-\nu}\right\|\right| X B\right\|\right|^{\nu}}\right)^{\frac{\tau(1-\tau)}{\nu(1-\nu)}} \\
& \leq\left\|A^{1-\tau} X B^{\tau}\right\| \mid\left(\frac{\||A X\||\||X B \||}{\left\|\left|A^{\frac{1}{2}} X B^{\frac{1}{2}} \|\right|^{2}\right.}\right)^{R(\tau)}\left(\frac{(1-\nu)\||A X\||+\nu\||X B \||}{\left\|\left|A^{1-\nu} X B^{\nu} \|\right|\right.}\right)^{\frac{\tau(1-\tau)}{\nu(1-\nu)}} .
\end{aligned}
$$


On the other hand, if $(\||X B\||-\||A X \||)(\tau-\nu)<0$, then

$$
\begin{aligned}
& \left\|\left|A^{1-\tau} X B^{\tau} \|\right|\left(\frac{\left.\left\|A^{\frac{1}{2}} X B^{\frac{1}{2}}\right\|\right|^{2}}{\||A X\||\||X B \||}\right)^{R(\nu)}\left(\frac{\||(1-\nu) A X+\nu X B \||}{\left\|A^{1-\nu} X B^{\nu}\right\| \mid}\right)^{\frac{\tau(1-\tau)}{\nu(1-\nu)}}\right. \\
& \leq\left.\left\|\left|A X\left\|\left.\right|^{1-\tau}\right\|\right| X B\right\|\right|^{\tau}\left(\frac{(1-\nu)\||A X\||+\nu\||X B \||}{\left.\left\|\left|A X\left\|\left.\right|^{1-\nu}\right\|\right| X B\right\|\right|^{\nu}}\right)^{\frac{\tau(1-\tau)}{\nu(1-\nu)}} \\
& \leq(1-\tau)\||A X\||+\tau\||X B \|| \\
& \leq\|(1-\tau) A X+\tau X B\| \mid+R(\tau)(\||A X\||+\|X B\||-\|| A X+X B\| \mid) .
\end{aligned}
$$

Proof. When $(\||X B\||-\||A X \||)(\tau-\nu)>0$, the first inequality follows immediately because $\||\||$ is a norm. The second inequality follows from Corollary 2.7 on replacing $(a, b)$ by $(\||A X\||,\||X B \||)$. Then the third inequality follows from (2.5) and the fact that $\left\|A^{1-\nu} X B^{\nu}\right\| \leq \leq\left.\|A X\|\right|^{1-\nu}\left\||X B \||^{\nu}\right.$.

Now when $(\||X B\||-\||A X \||)(\tau-\nu)<0$, we apply Corollary 2.7, (2.4), (2.5) and (2.6) to obtain the desired inequalities.

\section{References}

[1] Bakherad, M., Krinć, M., Moslehian, Sal, M., Reverses of the Young inequality for matrices and operators, Rocky Mountain Journal of Mathematics, 46(2016), 1089-1105.

[2] Hirzallah, O., Kittaneh, F., Matrix Young inequalities for the Hilbert-Schmidt norm, Linear Algebra Appl., 308(2000), 77-84.

[3] Horn, F., Johnson, C., Matrix Analysis, Cambridge Univ. Press, 1985.

[4] Kittaneh, F., Norm inequalities for fractional powers of positive operators, Lett. Math. Phys., 27(1993), 279-285.

[5] Kittaneh, F., Manasrah, Y., Improved Young and Heinz inequalities for matrices, J. Math. Anal. Appl., 36(2010), 262-269.

[6] Kittaneh, F., Manasrah, Y., Reverse Young and Heinz inequalities for matrices, Linear Multilinear Algebra, 59(2011), 1031-1037.

[7] Kittaneh, F., Moslehian, M., Sababbeh. M., Quadratic interpretation of the Heinz means, Math. Ineq. Appl. (in press).

[8] Krnić, M., More accurate Young, Heinz, and Hölder inequalities for matrices, Period. Math. Hung., 71(2015), 78-91.

[9] Liao, W., Wu, J., Reverse arithmetic-harmonic mean and mixed mean operator inequalities, J. Inequal. Appl., 2015:215.

[10] Mitroi, F., About the precision in Jensen-Steffensen inequality, Annals of the University of Craiova, 37(2010), 73-84.

[11] Sababheh, M., Log and harmonically log-convex functions related to matrix norms, Operators and Matrices, 10(2016), 453-465.

[12] Sababheh, M., Convex functions and means of matrices, Math. Ineq. Appl., 20(2017), $29-47$.

[13] Sababheh, M., Means refinements via convexity, Mediterranean Journal of Math., (2017), 14-125. 
[14] Sababheh, M., Choi, D., A complete refinement of Young's inequality, J. Math. Anal. Appl., 440(2016), 379-393.

[15] Sababheh, M., Moslehian, Sal, M., Advanced refinements of Young and Heinz inequalities, Journal of Number Theory, 172(2017), 178-199.

[16] Zhao, J., Wu, J., Operator inequalities involving improved Young and its reverse inequalities, J. Math. Anal. Appl., 421(2015), 1779-1789.

[17] Zuo, H., Shi, G., Fujii, M., Refined Young inequality with Kantorovich constant, J. Math. Inequal., 5(2011), 551-556.

Mohammad Sababheh

Department of Basic Sciences

Princess Sumaya University for Technology

Al Jubaiha, Amman 11941, Jordan

e-mail: sababheh@psut.edu.jo, sababheh@yahoo.com

Department of Mathematics

University of Sharjah

Sharjah 27272, UAE

e-mail: msababheh@sharjah.ac.ae 